0009-2509(94)00183-9

\title{
A more strict criterion for application of homogeneous models in modelling wall-cooled packed-bed reactors
}

(First received 16 July 1993; accepted in revised form 19 May 1994)

Recently, Wijngaarden and Westerterp (1989) derived a dimensionless criterion as to whether or not a homogeneous model can be used instead of a heterogeneous one in modelling wall-cooled tubular reactors. Their work is an extension of the well-known criteria of e.g. Mears (1971) for the neglect of interparticle mass and heat transfer resistances. Important, but somewhat restrictive, assumptions in their article are the linearization of the reaction rate with respect to the coolant temperature and the neglect of an external mass transfer limitation between the catalyst pellets and the gas phase. In this short communication we will demonstrate that simple extensions of their criterion, considerably enlarging its applicability, can be derived. Wijngaarden and Westerterp introduced a dimensionless number $\zeta$ :

$$
\zeta=\frac{\left(-\Delta H_{r}\right) V_{p}}{(1-\varepsilon) \alpha_{p} A_{p}}\left[\frac{\partial R(T)}{\partial T}\right]_{T=T_{r}}=\frac{R\left(T_{k}\right)-R\left(T_{q}\right)}{R\left(T_{k}\right)} .
$$

Therefore $\zeta$ is a measure of the relative increase in reaction rate due to external heat transfer resistance between the gas phase and the catalyst particles. The derivation is based on the following linearization of the reaction rate at the catalyst temperature:

$$
R\left(T_{k}\right)=R\left(T_{c}\right)+\left(T_{k}-T_{c}\right)\left[\frac{\partial R(T)}{\partial T}\right]_{T=T_{e}} .
$$

The temperature difference between the centre of the reactor tube and the coolant temperature can be easily $20^{\circ} \mathrm{C}$ or more. Because the reaction rate is exponentially dependent on temperature, it is clear that this linearization might severely underestimate the reaction rate at $T_{a}$ and $T_{k}$.

Figure 1 shows two alternative approximations to estimate the reaction rate at the catalyst temperature, depending on the linearization of the reaction rate as a function of temperature. The linearization based on the gas temperature, which is usually measured [see Wijngaarden and Westerterp (1993)], is preferred above the extrapolation based on $\vec{T}_{c}$ and $T_{g^{*}}$.

Using the linear extrapolation 3 and accounting for mass transfer, $R\left(T_{k}, C_{k}\right)$ can be written as

$$
\begin{aligned}
R\left(T_{k}, C_{k}\right)= & R\left(T_{g}, C_{g}\right)+\left(T_{k}-T_{g}\right)\left[\frac{\partial R\left(T, C_{g}\right)}{\partial T}\right]_{T=T_{g}} \\
& +\left(C_{k}-C_{g}\right)\left[\frac{\partial R\left(T_{g}, C\right)}{\partial C}\right\rceil_{C=C_{k}} .
\end{aligned}
$$

Combining this equation with the steady-state heat balance and the steady-state equation for external mass transfer,

$$
\begin{aligned}
& \alpha_{p} A_{p}\left(T_{k}-T_{g}\right)=\frac{V_{p} R\left(T_{k}\right)\left(-\Delta H_{r}\right)}{(1-\varepsilon)} \\
& R\left(T_{k}, C_{k}\right)=k_{g} \frac{A_{p}}{V_{p}}\left(C_{g}-C_{k}\right)(1-\varepsilon)
\end{aligned}
$$

the next relation can be derived:
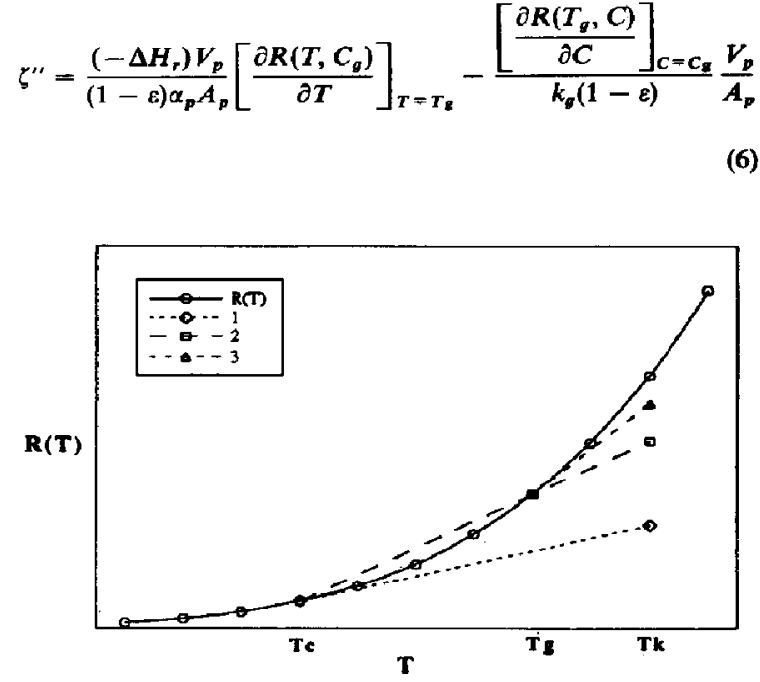

Fig. 1. Different approximations of the reaction rate at the catalyst temperature: (1) linearization based on $T_{c}$ [applied by Wijngaarden and Westerterp, eq. (1)]; (2) linear extrapolation based on $T_{c}$ and $T_{a}: R\left(T_{k}\right)=R\left(T_{g}\right)+\left[R\left(T_{g}\right)\right.$ $\left.-R\left(T_{c}\right)\right]\left(T_{k}-T_{g}\right) /\left(T_{g}-T_{c}\right)$; (3) linearization based on $T_{g}$ [eq. (3)].

where $\xi^{\prime \prime}$ is a measure of the relative increase in reaction rate due to external heat and mass transfer resistances. The first term of eq. (6) refers to heat transfer and the second term to mass transfer. The latter term can be neglected if the external mass transfer is negligible or if, for example, the mass transfer is lumped in the description of $R\left(T_{g}, C_{g}\right)$. From Fig. 1 it is clear that $\zeta^{\prime \prime}>\zeta$ if mass transfer limitation is neglected. The new criterion becomes

$$
\xi^{\prime \prime}<0.10
$$

This criterion will be applied in two cases concerning wall-cooled tubular reactors, already published in the literature. The first study was published by Vortmeyer and Haidegger (1991) and concerns the oxidation of ethane. Because mass transfer has been lumped in the description of the kinetics of this reaction, $\zeta^{\prime \prime}$ can only be applied without the last term referring to mass transfer limitation.

Figure 2 presents $\zeta$ and $\zeta^{\prime \prime}$ as a function of the gas temperature for their experiments from case $3 \mathrm{~d}$, using the ethane fraction measured near the hot spot. From this figure it is evident that $\zeta$ " should be used. The gas-phase temperature should not exceed $660 \mathrm{~K}$ near this hot spot (the actual 


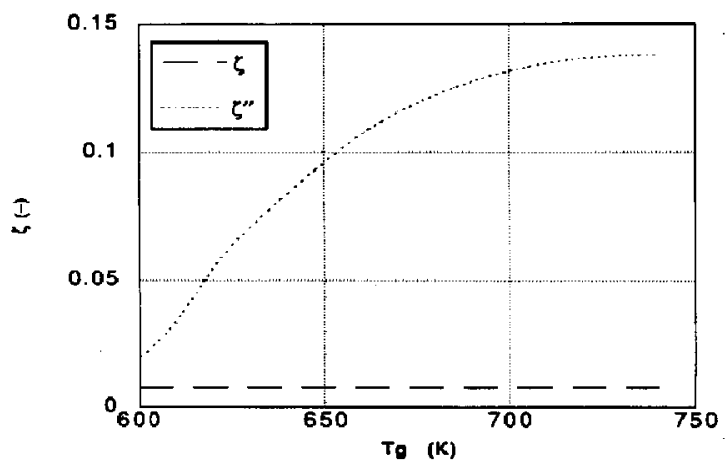

Fig. 2. Different parameters for discrimination between homogeneous and heterogeneous models in ethane oxidation [Vortmeyer and Haidegger (1991), case 3d, $y=0.0018$, $N u_{p}=10$; Agarwal (1988)].

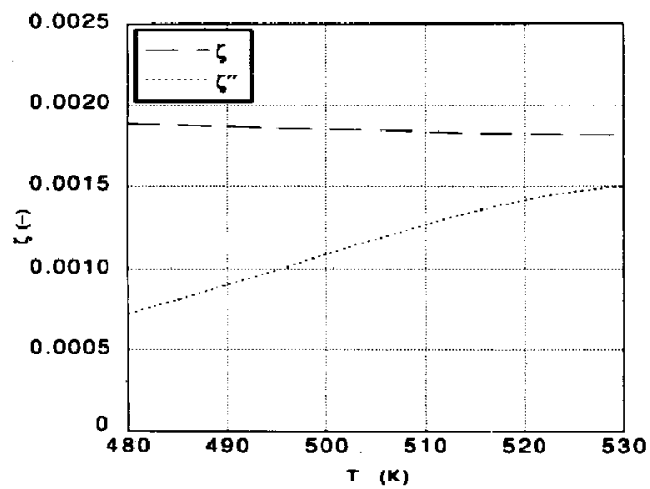

Fig. 3. Different parameters for discrimination between homogeneous and heterogeneous models in selective oxidation of ethylene (Borman and Westerterp, 1992).

measured temperature was about $670 \mathrm{~K}$ ). Other data in the same paper lead to much higher values of $\zeta "$, so these data should have been interpreted with a heterogeneous model; see also Westerterp et al. (1993).

The second study was published by Borman and Westerterp (1992). The reaction involved is the epoxidation of ethylene. Figure 3 presents $\zeta$ and $\zeta^{\prime \prime}$ as a function of relevant gas-phase temperatures. The reaction kinetics have been correlated for the indicated temperature range. Mass transfer has not been lumped into the kinetics. In this figure $\zeta^{\prime \prime}$ is very small and close to zero. Due to the inclusion of mass transfer resistance, $\zeta^{\prime \prime}$ is even smaller than $\zeta$. High catalyst activity in combination with a low heat of reaction might cause $\zeta$ " to become negative. The conclusion in the case of the experi- ments of Borman and Westerterp (1992) is that a homogeneous model can be applied without underestimating the reaction rate.

K. R. WESTERTERP ${ }^{\dagger}$ W. DE JONG

University of Twente G. H. W. VAN BENTHEM Department of Chemical Engineering PO Box 217, 7500 AE Enschede The Netherlands

\section{NOTATION}

$A_{p} \quad$ external surface area of catalyst pellet, $\mathrm{m}^{2}$ $C_{k} \quad$ concentration at the particle surface, $\mathrm{mol} / \mathrm{m}^{3}$ $C_{g} \quad$ concentration in the gas phase, $\mathrm{mol} / \mathrm{m}^{3}$

$\Delta H_{r} \quad$ reaction enthalpy, $\mathrm{kJ} / \mathrm{mol}$

$k_{g} \quad$ gas-solid mass transfer coefficient, $\mathrm{m} / \mathrm{s}$

$N u_{p} \quad$ Nusselt number, dimensionless

$R(T, C)$ reaction rate, $\mathrm{mol} / \mathrm{m}^{3} \mathrm{~s}$

$R$ gas constant $(=8.3143), J / m o l ~ K$

$T_{c} \quad$ coolant temperature, $\mathrm{K}$

$T_{g} \quad$ gas temperature, $\mathrm{K}$

$T_{k} \quad$ catalyst temperature, $K$

$V_{p} \quad$ volume of catalyst pellet, $\mathrm{m}^{3}$

Greek letters

$\alpha_{p} \quad$ heat transfer coefficient between particle and gas, $W /\left(\mathbf{m}^{2} \mathbf{K}\right)$

$\varepsilon$ volume fraction of gas in the catalyst bed, dimensionless

$\zeta, \zeta^{\prime \prime}$ dimensionless numbers [see eqs (1) and (6)]

\section{REFERENCES}

Agarwal, P. K., 1988, Transport phenomena in multi-particle systems-II. Particle-fluid heat and mass transfer. Chem. Engng Sci. 43, 2501-2510.

Borman, P. C. and Westerterp, K. R., 1992, An experimental study of the selective oxidation of ethylene in a wall cooled tubular packed bed reactor. Chem. Engng Sci. 47, 2541-2546.

Mears, D. E., 1971, Tests for transport limitations in experimental catalytic reactors. Ind. Engng Chem. Process Des. Dev. 10, 541-547.

Vortmeyer, D. and Haidegger, E., 1991, Discrimination of three approaches to evaluate heat fluxes from wall-cooled fixed bed chemical reactors. Chem. Engng Sci. 46, 2651-2660.

Westerterp, K. R., de Jong, W. and van Benthem, B. H. W., 1993, Comments on discrimination of three approaches to evaluate heat fluxes for wall-cooled fixed bed chemical reactors, Chem. Engng Sci. 48, 2669-2673.

Wijngaarden, R. J. and Westerterp, $K$. $R$, 1989, Do the effective heat conductivity and the heat transfer coefficient at the wall inside a packed bed depend on a chemical reaction? Weaknesses and applicability of current models. Chem. Engng Sci. 44, 1653-1663.

\footnotetext{
${ }^{\dagger}$ Author to whom correspondence should be addressed.
} 